\title{
Building DEM for deep open-pit coal mines using DJI Inspire 2
}

Nghia Viet Nguyen *

Faculty of Geomatics and Land Administration, Hanoi University of Mining and Geology, Vietnam

\begin{tabular}{l} 
ARTICLE INFO \\
\hline Article history: \\
Received 15th Nov 2019 \\
Revised 6 ${ }^{\text {th }}$ Jan. 2020 \\
Accepted 28 ${ }^{\text {th }}$ Feb. 2020 \\
\hline Keywords: \\
Deep open-pit mine, \\
Digital Elevation Model, \\
Inspire 2, \\
Unmanned aerial vehicle.
\end{tabular}

ARTICLE INFO

ABSTRACT

Using photo data of unmanned aerial vehicle (UAV) for building $3 D$ models has been widely used in recent years. However, building a 3D model for deep open - pit coal mines with the mean height difference between surface and bottom of mines to over $500 \mathrm{~m}$, there has not been researched mentioned. The paper deals with the assessment possibility of developing 3D models for deep open - pit mines from UAV image data. To accomplish this goal, DJI's Inspire 2 flying device is used to take the photo at Coc Sau coal mine. The flying area is $4 \mathrm{~km}^{2}$, the flight altitude compared to the takeoff point on the mine surface is $250 \mathrm{~m}$, the overlaying coverage is both horizontal and vertical is $70 \%$. The average errors of the horizontal and height elements of the reference points photo correlates are $0.011 \mathrm{~m}, 0.017 \mathrm{~m}, 0.016 \mathrm{~m}, 0.049 \mathrm{~m}$, and $0.051 \mathrm{~m}$. The maximum error on the $X$-axis is - 0,025 $\mathrm{m}$, and the $Y$-axis is $0.028 \mathrm{~m}$, the maximum horizontal error is $0.034 \mathrm{~m}$, the maximum error on the Z-axis is $0.095 \mathrm{~m}$, and the position error is $0.095 \mathrm{~m}$. These results show that the $3 \mathrm{D}$ model established from photographic data by Inspire 2 device has satisfied the requirements of the accuracy of establishing the mining terrain map 1: 1000 scale. 


\title{
Tạp chí Khoa học Kỹ thuật Mỏ - Địa chất
}

Trang điện tử: http://tapchi.humg.edu.vn

\section{Xây dựng mô hình số độ cao cho mỏ lộ thiên có độ sâu lớn từ dữ liệu ảnh chụp bằng thiết bị bay Inspire 2}

\author{
Nguyễn Viết Nghĩa
}

Khoa Trắc địa - Bản đồ và Quản lý đất đai, Trường Đại học Mỏ - Địa chất, Việt Nam

\begin{tabular}{|c|c|}
\hline THÔNG TIN BÀI BÁO & TÓM TẮT \\
\hline $\begin{array}{l}\text { Quá trình: } \\
\text { Nhận bài 15/11/2019 } \\
\text { Sưa xong 06/01/2020 } \\
\text { Chấp nhận đăng 28/02/2020 } \\
\text { Tù khóa: } \\
\text { Inspire 2, } \\
\text { Máy bay không người lái, } \\
\text { Mô hình số độ cao, } \\
\text { Mỏ lộ thiên sâu. }\end{array}$ & 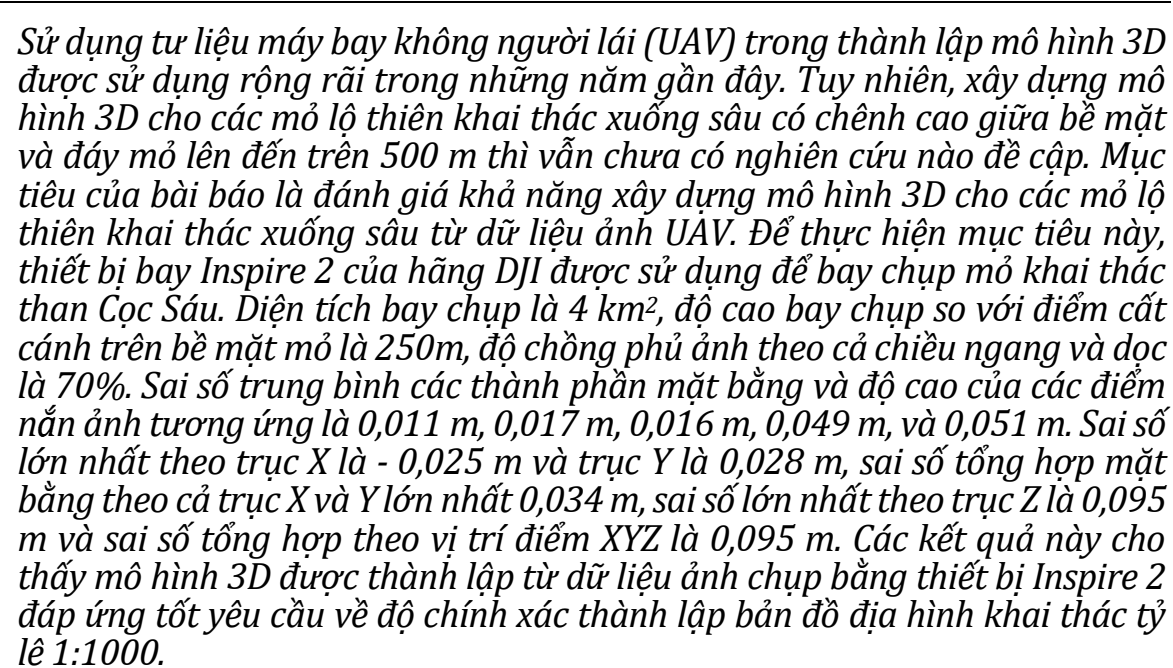 \\
\hline
\end{tabular}

(C) 2020 Trường Đại học Mỏ - Địa chất. Tất cả các quyền được bảo đảm.

\section{Mở đầu}

Mô hình 3D là dữ liệu quan trọng trong nhiều lĩnh vực. Mô hình này thường được thành lập bằng nhiều công nghệ khác nhau như đo đạc trực tiếp bằng máy toàn đạc điện tử, định vị vệ tinh GNSS, quét laser mặt đất và công nghệ bay quét - Lidar (Stal, Nuttens et al., 2011, Stempfhuber 2013, Beumier Charles and Mahamadou 2016). Tuy

*Tác giả liên hệ

E - mail: nguyenvietnghia@humg. edu.vn DOI: 10.46326/JMES.2020.61(1).01 nhiên, các công nghệ này có các nhược điểm như giá thành thiết bị cao, quá trình đo đạc tiêu tốn thời gian và sức lao động. Khó thực hiện trong các điều kiện địa hình và môi trường phức tạp và có thể gây mất an toàn lao động (Bùi Tiến Diệu và nnk., 2016). Ngày nay, sự xuất hiện của các thiết bị bay không người lái thực sự là cuộc cách mạng giúp cho công tác khảo sát đo vẽ thành lập bản đồ, giám sát và theo dõi sự biến động các thành phần và các đối tượng trên bề mặt Trái đất ngày càng hiệu quả (Razi et al., 2018). Trên thế giới, đã có nhiều nghiên cứu ứng dụng UAV trong xây dựng mô hình 3D các công trình (Irschara et al., 2010, 
Nex and Remondino, 2014) và trong đo đạc địa hình và công trình (Maza et al., 2011, Liu et al., 2014, Sona Giovanna et al., 2014).

Ở Việt Nam, việc ứng dụng công nghệ bay chụp UAV trong công tác xây dựng mô hình số bề mặt (Digital Surface Model - DSM) và bản đồ địa hình đã được thực hiện trong một số nghiên cứu. Máy bay không người lái MD - 1000 đã được Xí nghiệp bay chụp ảnh hàng không - Cục bản đồ Bộ tổng tham mưu sử dụng trong công tác thành lập bản đồ 3D cho khu vực Mỹ Đình và Thái Nguyên (Xí nghiệp bay chụp ảnh hàng không, 2011). Năm 2014, các tác giả Vũ Phan Long \& Lê Thắng thông qua kết quả thử nghiệm ứng dụng kết hợp giữa hệ thống UAV Swinglet CAM (Sensfly - Thụy sỹ) và UX5 (Trimble - Mỹ) trong thành lập bản đồ 3D hành lang tuyến điện, đã đưa ra khẳng định rằng: UAV hoàn toàn có thể sử dụng trong giám sát phạm vi hẹp, lập bản đồ 3D và thành lập bản đồ địa hình 1:2000 (Vũ Phan Long và Lê Thắng, 2014). Trong một nghiên cứu năm 2016, tác giả Bùi Tiến Diệu và đồng nghiệp đã xây dựng quy trình công nghệ sử dụng ảnh máy bay không người lái, xây dựng các sản phẩm bản đồ (mô hình số bề mặt, mô hình số độ cao, bản đồ trực ảnh, bản đồ 3D) (Bùi Tiến Diệu và nnk., 2016). Các tác giả Bùi Ngọc Quý và Phạm Văn Hiệp cũng đã thành lập bản đồ $3 \mathrm{D}$ bằng công nghệ UAV khu vực đồng bằng (Bùi Ngọc Quý, Phạm Văn Hiệp, 2017). Tác giả Mai Văn Sỹ và cộng sự đã nghiên cứu thành lập bản đồ tỷ lệ lớn từ dữ liệu ảnh bay chụp UAV (Mai Văn Sỹ và nnk., 2017). Tuy nhiên, các nghiên cứu này chỉ giới hạn trong khu vực có điều kiện địa hình đơn giản và bằng phẳng.

Các nghiên cứu ứng dụng UAV trong thành lập bản đồ khai thác mỏ ở Việt Nam chưa nhiều. Năm 2014 các tác giả Võ Chí Mỹ, Robert Duda đã thử nghiệm ứng dụng UAV UX - 5 của hãng Trimble trong đo vẽ khu vực mỏ lộ thiên và giám sát sự biến động môi trường mỏ (Võ Chí Mỹ, Robert Dudek, 2015). Trong một nghiên cứu khác của tác giả Bùi Tiến Diệu và các cộng sự năm 2017, thiết bị UAV được sử dụng bay chụp xây dựng mô hình số bề mặt khu vực bờ mỏ lộ thiên (Dieu Tien Bui và nnk., 2017). Tuy nhiên trong nghiên cứu này, bên cạnh các đánh giá về độ chính xác khi thay đổi đồ hình và số điểm khống chế ảnh, các tác giả chưa khảo sát độ cao bay chụp, chưa đưa ra được một quy trình bay chụp đầy đủ và rõ ràng cho mỏ lộ thiên có kích thước và công nghệ khai thác khác nhau. Bên cạnh đó, với đặc điểm địa hình biến động nhanh và lớn ở các mỏ lộ thiên, khả năng ứng dụng UAV cần được đánh giá chi tiết và toàn diện. Đầu năm 2019, tác giả Nguyễn Quốc Long đã ứng dụng công nghệ UAV trong xây dựng mô hình số bề mặt và bản đồ địa hình mỏ lộ thiên khai thác vật liệu xây dựng. Kết quả thực nghiệm cho độ chính xác đạt yêu cầu thành lập bản đồ tỷ lệ lớn. Từ nghiên cứu này, các tác giả đã kết luận UAV có qui trình bay chụp đơn giản, an toàn khi đo đạc tại các vùng có địa hình phức tạp (Nguyễn Quốc Long, Cao Xuân Cường, 2019). Trong một nghiên cứu khác (Nguyen Quoc Long et al., 2019), nhóm tác giả đã đánh giá khả năng sử dụng của máy bay không người lái giá rẻ trong việc xây dựng mô hình số địa hình và bản đồ, thực nghiệm được tiến hành tại 4 mỏ khai thác đá có đặc điểm địa hình khác nhau, trong cả bốn trường hợp đều sử dụng máy bay Phantom 4 Pro.

Hiện nay, vẫn chưa có một nghiên cứu nào về ứng dụng công nghệ này trong thành lập DEM ở các mỏ lộ thiên khai thác sâu, chênh cao địa hình trên bề mặt mỏ và đáy moong lớn tại Việt Nam. Do đó, mục tiêu chính của nghiên cứu này là đánh giá độ chính xác DEM thành lập từ phương pháp đo ảnh máy bay không người lái UAV cho địa hình mỏ lộ thiên khai thác sâu.

\section{Thiết bị bay và phần mềm xử lý}

\subsection{Thiết bị bay Inspire 2}

Trọng bộ thiết bị Inspire 2 bao gồm thân máy và bộ điều khiển. Các bộ phận chính gắn trên thân máy bao gồm các cảm biến tránh va đập theo 5 hướng (trái, phải, trước, sau, dưới), bốn mô tơ, 4 cánh quạt có thể tháo rời, chân hạ cánh cố định phía bên dưới. Bộ điều khiển từ xa gồm các nút bấm điều khiển quá trình bay, điều khiển có tích hợp 2 ăng ten với 2 tần số: 2,4 và $5,8 \mathrm{GHz}$ (DJI, 2017), có cổng kết nối với máy tính bảng hoặc điện thoại thông minh để cài đặt các thông số bay chụp hoặc hình hiển thị ảnh chụp trực tiếp từ máy bay. Hình ảnh của bộ thiết bị Inspire 2 như Hình 1 .

\subsection{Bộ phận chụp ảnh (camera)}

Camera của Inspire 2 có thể tùy biến, có thể dùng các camera có cùng một kiểu ngàm kết nối với thân máy bay. Thông thường hay sử dụng 2 loại camera là Zenmuse $\mathrm{X} 4 \mathrm{~S}$ và Zenmuse X5S (Hình 2). Các loại camera này có thể chụp ảnh tĩnh 
với độ phân giải 24 MP ở định dạng JPEG hoặc DNG RAW. Có khả năng quay video với độ phân giải $4 \mathrm{~K}$. Cảm biến camera có kích thước lớn hơn các thế hệ trước nó nên trong điều kiện thiếu sáng cho ra ảnh/video chất lượng tốt hơn. Ống kính của camera có khẩu độ lớn nhất $\mathrm{F} / 2.8$, cho góc nhìn rộng 94 độ. DJI cho biết ống kính của camera được thiết kế đặc biệt để chống méo ở vùng rìa ảnh. Camera được nối với bộ chống rung 3 chiều giúp cho ảnh chụp ổn định (DJI, 2017). Các thông số chụp ảnh có thể đặt ở chế độ tự động hoặc điều khiển thông qua bộ điều khiển mặt đất.

\subsection{Phần mềm xử lý ảnh chụp từ UAV}

Hiện nay có nhiều phần mềm xử lý ảnh UAV khác nhau như Agisoft Photoscan, ENVI, Trimple Business Center, Erdas Leica Photogrammetry có độ sâu - $250 \mathrm{~m}$, chênh cao lên bề mặt mỏ là 500 $\mathrm{m}$, diện tích bay chụp là 400 ha. Có thể coi mỏ này
Suite, PhotoModeler Scanner, Pix4UAV Desktop,... Trong nghiên cứu này, nhóm tác giả sử dụng phần mềm Agisoft Photoscan phiên bản 1.42 để xử lý toàn bộ quy trình từ khớp ảnh, tạo đám mây điểm, xây dựng mô hình số bề mặt (DSM), và thành lập bình đồ ảnh. Theo kết quả nghiên cứu (Sona Giovanna, et al., 2014) thì đây là phần mềm xử lý ảnh UAV tốt nhất. Giao diện phần mềm Agisoft Photoscan như Hình 3.

\section{Thực nghiệm xây dựng mô hình DSM cho mỏ lộ thiên sâu}

\section{1. Địa điểm thục nghiệm bay chụp}

Phần thực nghiệm được tiến hành tại mỏ lộ thiên Cọc Sáu. Đây là mỏ lộ thiên khai thác than xuống sâu nhất tại Việt Nam, hiện tại đáy moong là các mỏ tiêu biểu cho các mỏ khai thác than xuống sâu tại Việt Nam.
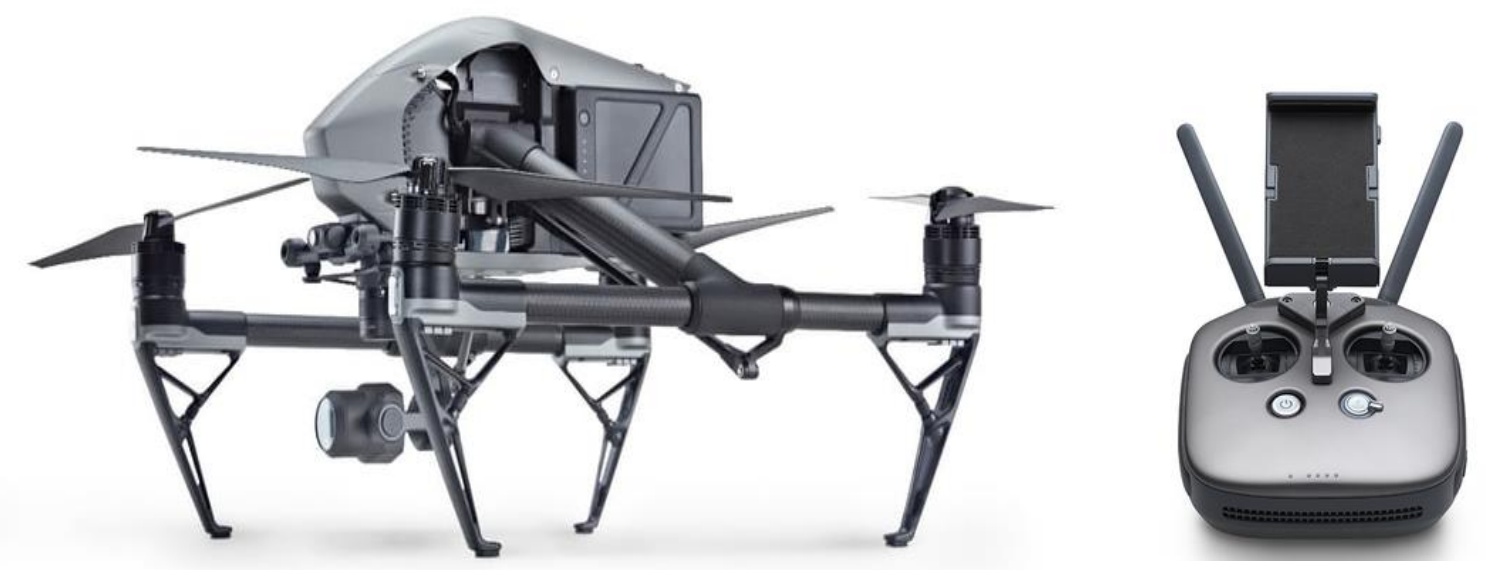

Hình 1. Bộ thiết bị bay Inspire 2.

(http://blog.geekbuying.com/2016/11/dji - inspire - 2 - release - date - price - and - specifications).

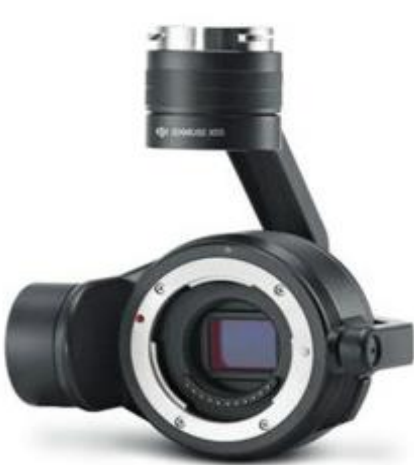

Zenmuse X4S

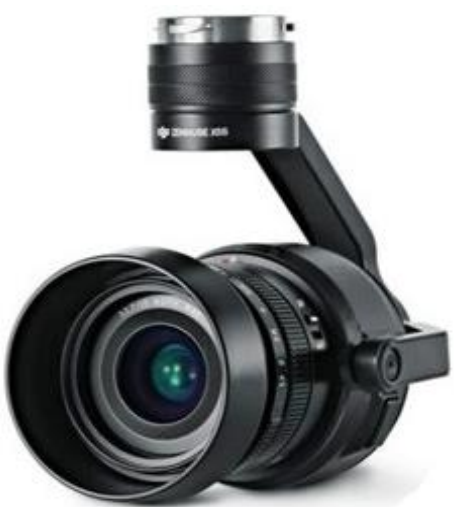

Zenmuse X5S

Hình 2. Camera của Inspire 2. 


\subsection{Xây dưng điểm khống chế và kiểm tra}

Điểm khống chế ảnh được đo đạc bằng công nghệ định vị vệ tinh (GNSS) xử lý thức thời (Real Time Kinematic - RTK). Những điểm này được sử dụng cho hai mục đích là nắn ảnh về hệ tọa độ VN - 2000 và đánh giá độ chính xác của mô hình DSM. Các tiêu đánh dấu điểm khống chế ảnh có đường kính $50 \mathrm{~cm}$, được làm bằng vật liệu phản chiếu cao để tăng cường độ tương phản, dễ dàng phát hiện trên ảnh (Hình 4).

Trong nghiên cứu này, tổng số điểm khống chế và kiểm tra là 35 điểm, trong đó 17 điểm dùng để nắn ảnh và 8 điểm còn lại dùng để đánh giá độ chính xác mô hình, các điểm dùng để đánh giá độ chính xác được phân bố đều trên khu vực bay chụp và nằm ở các độ cao khác nhau, đảm bảo tính khách quan trong đánh giá kết quả (Hình 5).

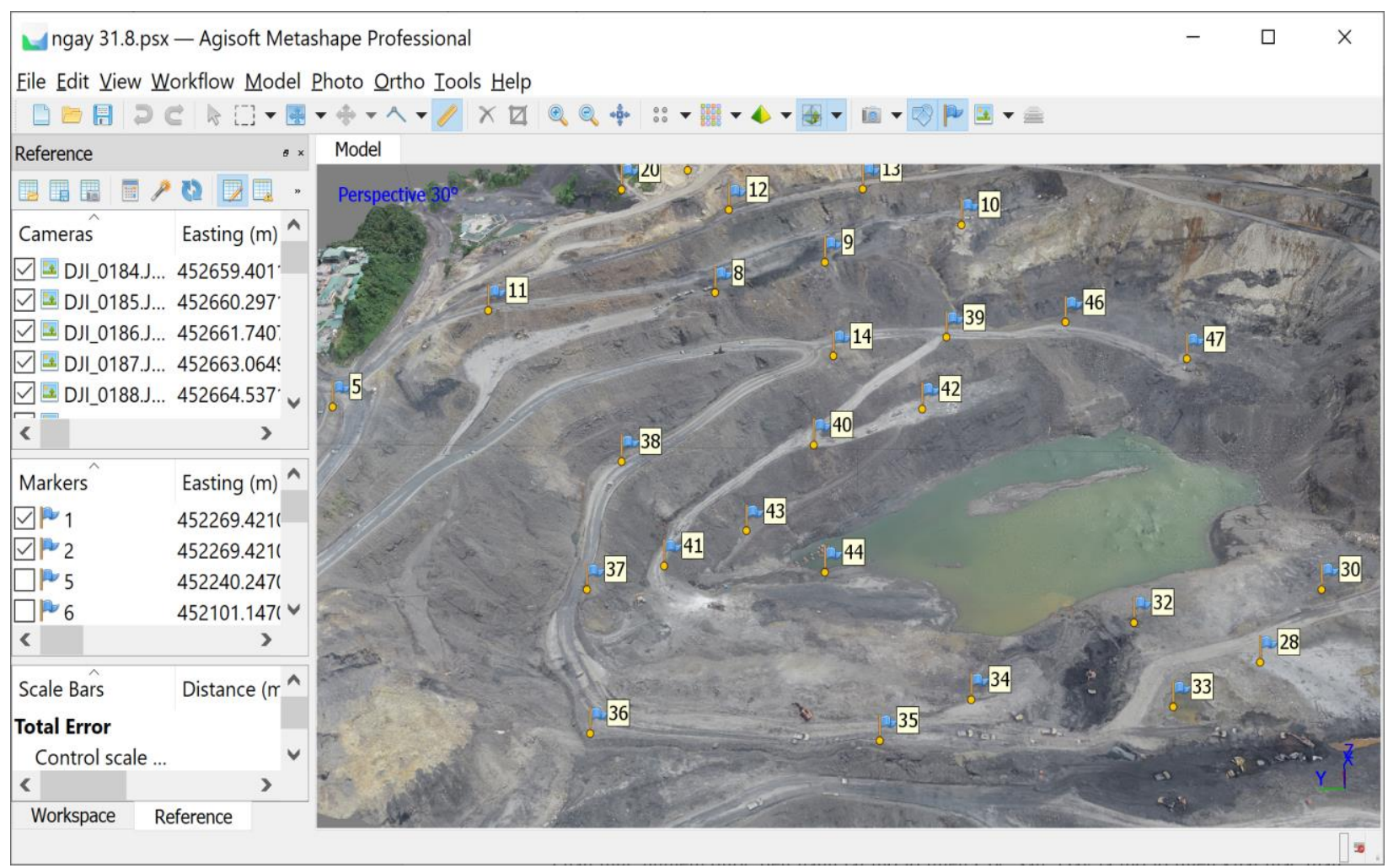

Hình 3. Giao diện phần mềm Agisoft Photoscan.
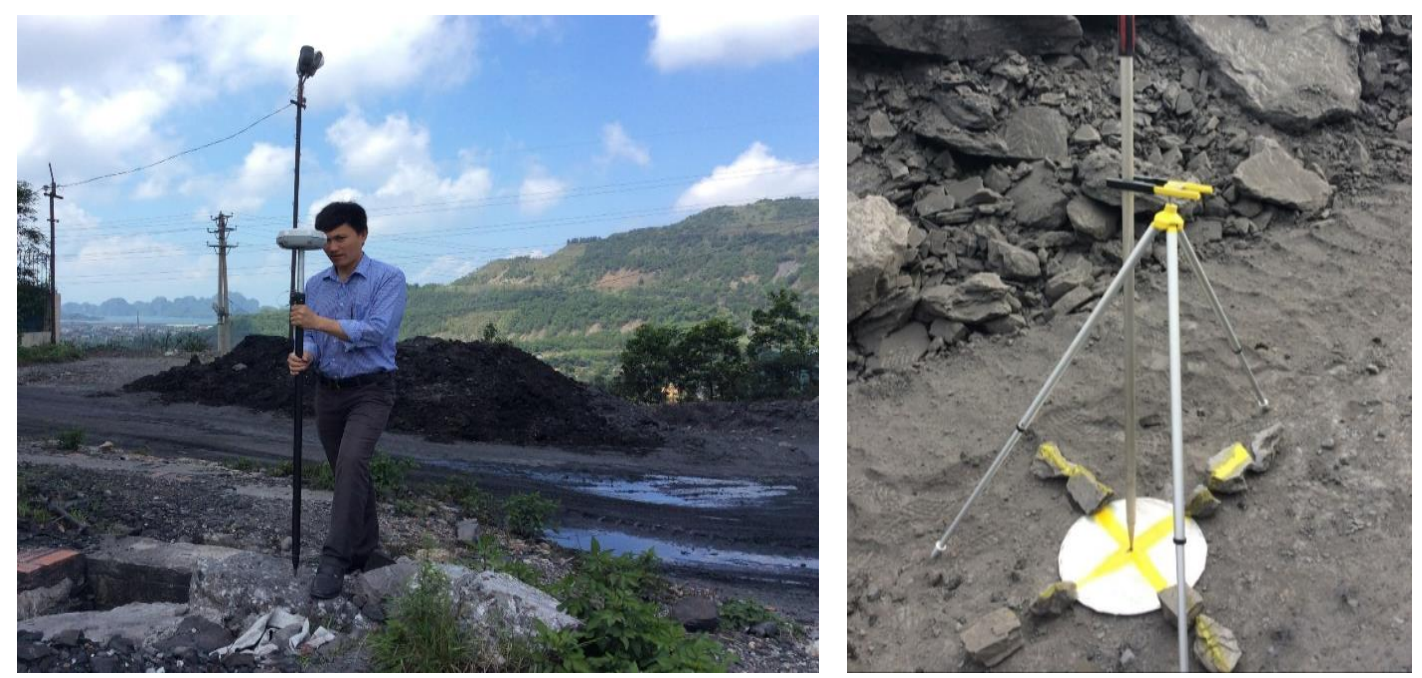

Hình 4. Thiết bị đo GNSS/RTK và tiêu đánh dấu điểm khống chế. 


\subsection{Thiết kế và thực hiện bay chụp}

Quá trình bay chụp được thiết kế trên phần mềm Pix4D Capture (Hình 6), có thể thiết kế độ cao bay, tốc độ bay, độ chồng phủ theo hướng dọc và ngang, góc chụp ảnh,... Khi cài đặt ở chế độ bay an toàn (Safe mode), UAV sẽ tự động bay theo các dải đã thiết kế bao gồm tự động cất cánh, bay lên độ cao thiết kế, sau đó bay đến các điểm thiết kế để dừng và chụp ảnh như đã thiết lập trong phần mềm. Khi ảnh cuối cùng được chụp xong, UAV sẽ tự động bay về và hạ cánh tại điểm xuất phát. Trong quá trình bay chụp, trạng thái hoạt động của UAV và máy ảnh được hiện thị liên tục trên máy tính bảng hoặc điện thoại thông minh. Tùy vào độ an toàn của UAV (ví dụ tình trạng năng lượng của pin, nguy cơ va chạm với vật thể, động vật trên không,...), người điều khiển có thể dừng quá trình bay tự động để điều khiển UAV hạ cánh. Để đảm bảo an toàn cho máy bay, tránh va đập vào các ngọn núi thì độ cao bay chụp được lựa chọn sao cho luôn cao hơn điểm cao nhất của khu vực

bay. Do hạn chế về dung lượng pin nên thời gian bay mỗi ca chỉ xấp xỉ 20 phút. Vì thế để hoàn thành được diện tích bay chụp lớn, cần thiết kế nhiều dải bay liên tiếp. Độ phủ ngang và phủ dọc của các tuyến bay thiết kế là 70\%.

\section{4. Đánh giá độ chính xác của mô hình số bề mặt (DSM)}

Độ chính xác của mô hình DSM mỏ được đánh giá trên cơ sở so sánh tọa độ và độ cao của các điểm trên mô hình với các điểm khống chế mặt đất, độ chính xác của mô hình 3D được đánh giá theo các công thức sau:

$$
\begin{gathered}
\Delta X=X_{D S M}-X_{C C P} \\
\Delta Y=Y_{D S M}-Y_{C C P} \\
\Delta Z=Z_{D S M}-Z_{C C P} \\
\Delta X Y Z=X Y Z_{D S M}-X Y Z_{C C P} \\
R M S E_{X}=S Q R T\left[(1 / n) \sum_{i=1}^{n}\left(X_{D S M}-X_{G C P i}\right)^{2}\right]
\end{gathered}
$$

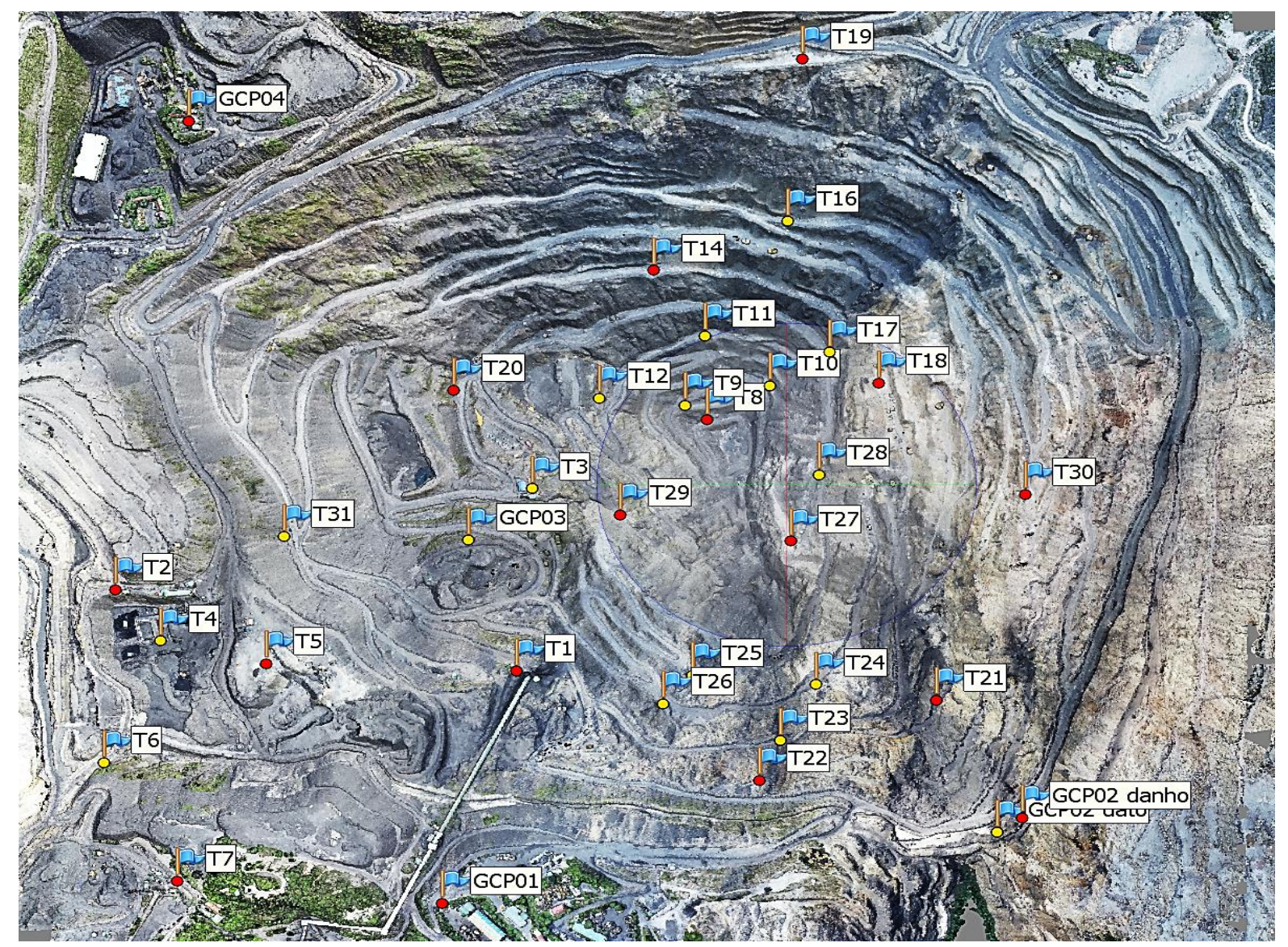

Hình 5. Phân bố điểm nắn ảnh và điểm đánh giá độ chính xác. 


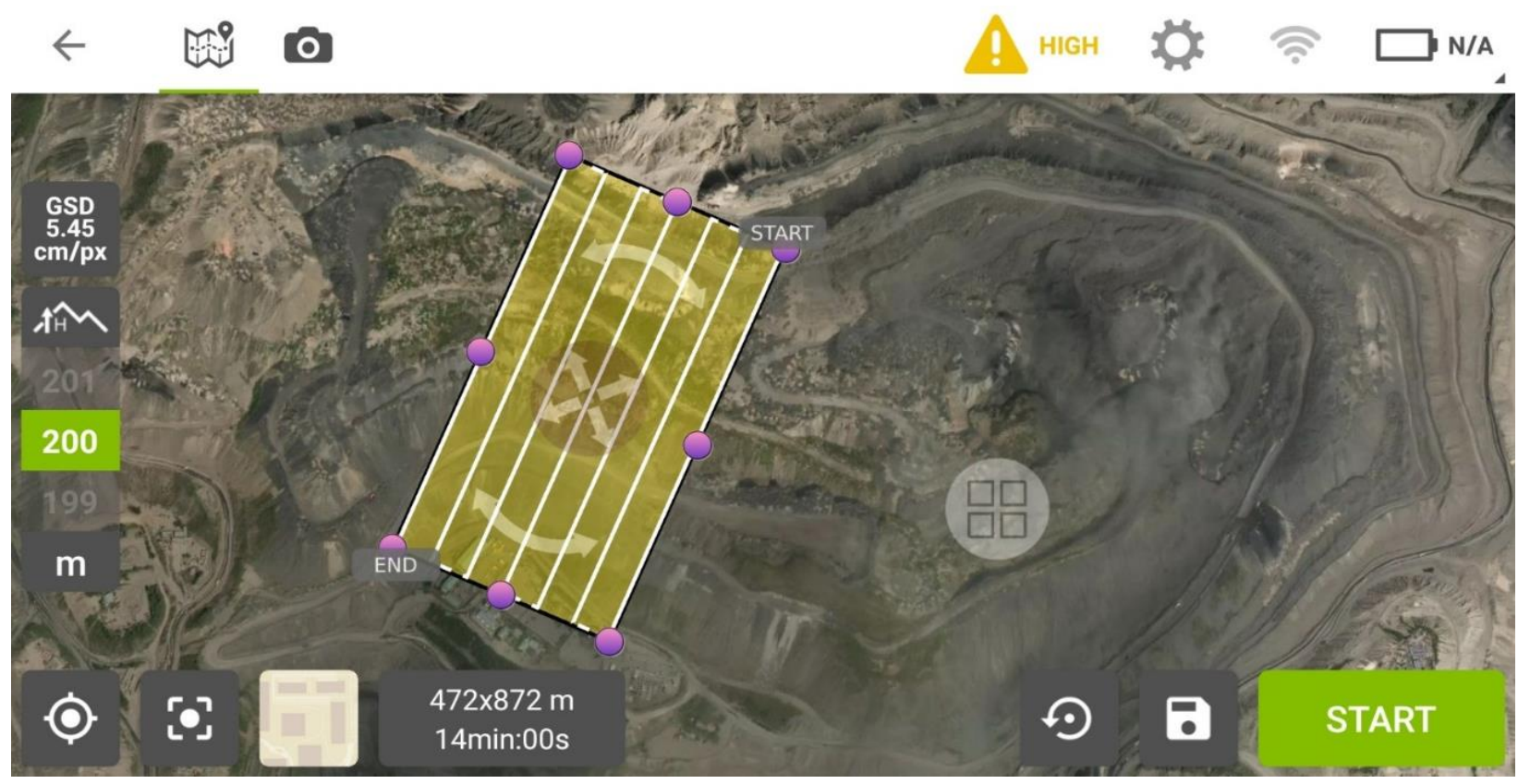

Hình 6. Thiết kế dải bay tại cụm mỏ Cọc Sáu.

$$
\left.\begin{array}{l}
R M S E_{Y}=S Q R T\left[(1 / n) \sum_{i=1}^{n}\left(Y_{D S M}-Y_{G C P i}\right)^{2}\right] \\
R M S E_{Z}=S Q R T\left[(1 / n) \sum_{i=1}^{n}\left(Z_{D S M}-Z_{G C P i}\right)^{2}\right] \\
R M S E_{X Y Z}= \\
\quad S Q R T\left\{( 1 / n ) \sum _ { i = 1 } ^ { n } \left[\left(X_{D S M}-X_{G C P i}\right)^{2}+\right.\right. \\
\left.\left.\quad\left(Y_{D S M}-Y_{G C P i}\right)^{2}+\left(Z_{D S M}-Z_{G C P i}\right)^{2}\right]\right\} .
\end{array}\right]
$$

Trong đó: $\Delta X, \Delta Y, \Delta X, \Delta Z, \Delta X Y Z$ - Các giá trị chênh lệch các thành phần tọa độ, độ cao và vị trí điểm; RMSE - Sai số trung phương; $\mathrm{n}$ tổng số điểm kiểm tra; $X_{G C P i}$ và $X_{D S M}, Y_{G C P i}$ và $Y_{D S M}, Z_{G C P i}$ và $Z_{D S M^{-}}$ Tương ứng là thành phần tọa độ theo trục $X$, trục $Y$ và trục $Z$ của điểm khống chế và mô hình DSM.

\section{Kết quả và thảo luận}

Sau khi xử lý ghép ảnh bằng phần mềm Agisoft Photoscan, sai số trung phương của tất cả các điểm nắn ảnh như trong Bảng 1. Cụ thể, sai số của các điểm dùng để nắn ảnh như sau: sai số trung bình các thành phần $\mathrm{X}, \mathrm{Y}, \mathrm{XY}, \mathrm{Z}, \mathrm{XYZ}$ tương ứng là $0,011 \mathrm{~m}, 0,017 \mathrm{~m}, 0.016 \mathrm{~m}, 0,049 \mathrm{~m}$ và $0,051 \mathrm{~m}$. Sai số lớn nhất theo trục $X$ là - $0,025 \mathrm{~m}$ và trục $Y$ là $0,028 \mathrm{~m}$, sai số tổng hợp mặt bằng theo cả trục $\mathrm{X}$ và $Y$ lớn nhất $0,034 \mathrm{~m}$, sai số lớn nhất theo trục $\mathrm{Z}$ là $0,095 \mathrm{~m}$ và sai số tổng hợp theo vị trí điểm XYZ là $0,095 \mathrm{~m}$.
Sai số trung phương của các thành phần $\mathrm{X}, \mathrm{Y}$, $\mathrm{XY}, \mathrm{Z}, \mathrm{XYZ}$ của tất cả các điểm kiểm tra độ chính xác mô hình số địa hình (DTM) tương ứng là 0,011 $\mathrm{m}, 0,017 \mathrm{~m}, 0,016 \mathrm{~m}, 0,049 \mathrm{~m}$, và $0,051 \mathrm{~m}$ (Bảng 2). Sai số lớn nhất theo trục $X$ là - $0,120 \mathrm{~m}$ và trục Y là $0,166 \mathrm{~m}$, sai số tổng hợp mặt bằng theo cả trục $X$ và Y lớn nhất $0,205 \mathrm{~m}$, sai số lớn nhất theo trục Z là $0,255 \mathrm{~m}$ và sai số tổng hợp theo vị trí điểm $X Y Z$ là $0,309 \mathrm{~m}$. Mô hình DTM của mỏ Cọc Sáu như trên Hình 7.

Từ kết quả đánh giá độ chính xác ở trên cho thấy mô hình DTM có độ trùng khớp cao với địa hình thực tế với mô hình 3D của mỏ Cọc Sáu như Hình 7. Theo qui phạm Việt Nam ngành Trắc địa mỏ (Bộ Công Thương 2015) và thông tư số 68/2015/TT - BTNMT (Bộ Tài Nguyên và Môi Trường 2015), độ chính xác của cả hai mô hình ở trên là đáp ứng được yêu cầu thành lập bản đồ địa hình tỷ lệ lớn 1:1000.

Từ mô hình 3D, sử dụng các phần mềm đồ họa như Autocad, Microstation để biên tập các bản đồ địa hình, bản đồ hiện trạng sử dụng đất của các mỏ,... Nhờ mô hình có độ phân giải cao, hình ảnh trực quan, chân thực, rõ nét và chính xác, quá trình biên tập bản đồ thuận lợi. 
Bảng 1. Tọa độ và độ cao các điểm nắn ảnh và độ chính xác.

\begin{tabular}{|c|c|c|c|c|c|}
\hline Tên điểm & Sai số $\Delta \mathrm{X}(\mathrm{m})$ & Sai số $\Delta \mathrm{Y}(\mathrm{m})$ & Sai số $\Delta \mathrm{XY}(\mathrm{m})$ & Sai số $\Delta \mathrm{Z}(\mathrm{m})$ & Sai số $\Delta \mathrm{XYZ}(\mathrm{m})$ \\
\hline GCP01 & 0,003 & 0,002 & 0,004 & 0,011 & 0,012 \\
\hline GCP02 danho & $-0,004$ & $-0,014$ & 0,015 & $-0,02$ & 0,058 \\
\hline GCP04 & $-0,012$ & 0,001 & 0,012 & 0,021 & 0,016 \\
\hline T2 & $-0,004$ & 0,013 & 0,014 & 0,013 & 0,019 \\
\hline T5 & 0,007 & 0,007 & 0,010 & $-0,016$ & 0,019 \\
\hline T7 & 0,005 & $-0,002$ & 0,005 & $-0,054$ & 0,054 \\
\hline T8 & $-0,02$ & $-0,028$ & 0,034 & $-0,063$ & 0,072 \\
\hline T14 & 0,015 & $-0,012$ & 0,019 & $-0,054$ & 0,057 \\
\hline T18 & 0,004 & $-0,007$ & 0,008 & $-0,025$ & 0,026 \\
\hline T19 & $-0,008$ & 0,001 & 0,008 & 0,095 & 0,095 \\
\hline T20 & $-0,007$ & $-0,003$ & 0,008 & $-0,014$ & 0,016 \\
\hline T21 & $-0,007$ & $-0,011$ & 0,013 & 0,091 & 0,092 \\
\hline T22 & 0,006 & $-0,008$ & 0,010 & 0,004 & 0,011 \\
\hline T27 & $-0,025$ & $-0,017$ & 0,030 & 0,063 & 0,070 \\
\hline T29 & $-0,016$ & 0,012 & 0,020 & 0,079 & 0,081 \\
\hline T30 & $-0,007$ & $-0,013$ & 0,015 & $-0,028$ & 0,032 \\
\hline T1 & $-0,004$ & $-0,008$ & 0,009 & $-0,017$ & 0,019 \\
\hline RMSE & 0,011 & 0,017 & 0,016 & 0,049 & 0,051 \\
\hline
\end{tabular}

Bảng 2. Tọa độ và độ cao các điểm kiểm tra và độ chính xác.

\begin{tabular}{|c|c|c|c|c|c|}
\hline Tên điểm & Sai số $\Delta \mathrm{X}(\mathrm{m})$ & Sai số $\Delta \mathrm{Y}(\mathrm{m})$ & Sai số $\Delta \mathrm{XY}(\mathrm{m})$ & Sai số $\Delta \mathrm{Z}(\mathrm{m})$ & Sai số $\Delta \mathrm{XYZ}(\mathrm{m})$ \\
\hline GCP03 & 0,120 & 0,166 & 0,205 & $-0,195$ & 0,283 \\
\hline T3 & $-0,060$ & $-0,020$ & 0,063 & $-0,080$ & 0,102 \\
\hline T4 & $-0,098$ & 0,000 & 0,098 & 0,128 & 0,161 \\
\hline T6 & $-0,005$ & $-0,032$ & 0,032 & $-0,207$ & 0,210 \\
\hline T9 & 0,053 & $-0,003$ & 0,053 & $-0,204$ & 0,309 \\
\hline T10 & 0,069 & 0,037 & 0,078 & 0,255 & 0,267 \\
\hline T11 & 0,094 & $-0,094$ & 0,133 & 0,148 & 0,199 \\
\hline T12 & 0,022 & 0,003 & 0,022 & $-0,148$ & 0,150 \\
\hline T15 & 0,035 & $-0,011$ & 0,037 & 0,090 & 0,097 \\
\hline T16 & 0,044 & $-0,032$ & 0,054 & $-0,035$ & 0,065 \\
\hline T17 & 0,033 & $-0,089$ & 0,095 & $-0,081$ & 0,125 \\
\hline T23 & $-0,016$ & $-0,064$ & 0,066 & $-0,075$ & 0,068 \\
\hline T24 & 0,006 & $-0,058$ & 0,058 & $-0,071$ & 0,059 \\
\hline T25 & 0,070 & 0,077 & 0,136 & 0,085 & 0,144 \\
\hline T26 & $-0,079$ & 0,002 & 0,079 & $-0,071$ & 0,079 \\
\hline T28 & $-0,108$ & $-0,017$ & 0,169 & 0,159 & 0,232 \\
\hline T31 & 0,082 & $-0,062$ & 0,128 & 0,068 & 0,145 \\
\hline GCP02 dato & 0,006 & 0,015 & 0,016 & 0,138 & 0,139 \\
\hline RMSE & 0,066 & 0,061 & 0,090 & 0,138 & 0,164 \\
\hline
\end{tabular}


Trong nghiên cứu này, độ chính xác đạt được có thể dùng để thành lập bản đồ tỷ lệ 1:1000. Trong các nghiên cứu tiếp theo, tác giả sẽ tập trung theo hướng điều chỉnh độ cao bay chụp, tăng tỷ lệ chồng phủ dọc và ngang, cũng như thay đổi góc chụp của camera đối với các sườn tầng dốc nhằm đạt được độ chính xác cao hơn.

\section{Tài liệu tham khảo}

Beumier Charles and I. Mahamadou, (2016). "Digital terrain models derived from digital surface model uniform regions in urban areas." International Journal of Remote Sensing 37(15): 3477 - 3493.

Bộ Công Thương, (2015). Tiêu chuẩn Việt Nam ngành Trắc Địa Mỏ.

Bộ Tài Nguyên và Môi Trường, (2015). Thông tư 68/2015/TT - BTNMT: Quy định kỹ thuật đo đạc trực triếp địa hình phục vụ thành lập bản đồ địa hình và cơ sở dữ liệu nền địa lý tỷ lệ 1:500, 1:1000, 1:2000, 1:5000.

Bùi Ngọc Quý, Phạm Văn Hiệp, (2017). Nghiên cứu xây dựng mô hình 3D từ dữ liệu ảnh máy bay không người lái (UAV). Tạp chí Khoa học kỹ thuật Mỏ - Địa chất 4 (58): 1 - 10.

Bùi Tiến Diệu, Nguyễn Cẩm Vân, Hoàng Mạnh Hùng, Đồng Bích Phương, Nhữ Việt Hà, Trần Trung Anh, Nguyễn Quang Minh, (2016). Xây dựng mô hình số bề mặt và bản đồ trực ảnh sử dụng công nghệ đo ảnh máy bay không người lái. Hội nghị khoa học: Đo đạc bản đồ với ứng phó biển đổi khí hậu, Hà Nội.

Dieu Tien Bui, Nguyen Quoc Long, Xuan - Nam Bui, Viet - Nghia Nguyen, Chung Van Pham, Canh Van Le, Phuong - Thao Thi Ngo, Dung Tien Bui, Bjørn Kristoffersen, (2017). Lightweight Unmanned Aerial Vehicle and Structure - from - Motion Photogrammetry for Generating Digital Surface Model for Open - Pit Coal Mine Area and Its Accuracy Assessment. International Conference on Geo - Spatial Technologies and Earth Resources: 17 - 33.

DJI, (2017). Phantom 4 Pro Visionary intelligence and elevated imagination, https://www.dji.com/phantom - 4 - pro.

http://blog.geekbuying.com/2016/11/dji - inspire - 2 - release - date - price - and specifications)

Irschara A., K. V., K. M., B. H. and L. F., (2010). Towards fully automatic photogrammetric reconstruction using digital images taken from uavs. 38: 65 - 70 .

Liu P., C. A. Y., H. Y., H. J., L. J., K. S., W. T., W. M. and T. M., (2014). A review of rotorcraft unmanned aerial vehicle (UAV) developments and applications in civil engineering. Smart Struct. Syst 13(6): 1065 - 1094.

Mai Văn Sỹ, Bùi Ngọc Quý, Phạm Văn Hiệp và Lê Đình Quý, (2017). Nghiên cứu xử dụng dữ liệu ảnh máy bay máy bay không người lái (UAV) trong thành lập bản đồ địa hình tỷ lệ lớn. Tạp chí khoa học Đo đạc và bản đồ 33 .

Maza I., C. F., C. J., M. - d. - D. J. R. and O. A., (2011). Experimental results in multi - UAV coordination for disaster management and civil security applications. Journal of intelligent \& robotic systems 61(1 - 4): 563 - 585.

Nex, F. and F. Remondino, (2014). UAV for 3D mapping applications: a review. Applied geomatics 6(1): 1 - 15.

Nguyễn Quốc Long and Cao Xuân Cường, (2019). Ứng dụng máy bay không người lái (UAV) để xây dựng mô hình số bề mặt và bản đồ mỏ lộ thiên khai thác vật liệu xây dựng. Tạp chí công nghiệp mỏ 1: 21 - 29.

Nguyen Quoc Long, Xuan - Nam Bui, Cao Xuan Cuong, Le Van Canh, (2019). An approach of mapping quarries in Vietnam using low - cost Unmanned Aerial Vehicles. International Journal of Sustainable Development 11(2):199 $-210$.

Razi, P., J. T. S. Sumantyo, D. Perissin, H. Kuze, M. Y. Chua and G. F. Panggabean, (2018). 3D land mapping and land deformation monitoring using persistent scatterer interferometry (PSI) ALOS PALSAR: Validated by geodetic GPS and UAV." IEEE Access 6: 12395 - 12404.

Sona Giovanna, P. Livio, P. Diana, P. Daniele and G. Rossana, (2014). Experimental analysis of different software packages for orientation and digital surface modelling from UAV 
images. Earth Science Informatics 7(2): 97 107.

Stal, C., T. Nuttens, J. Bourgeois, L. Carlier, P. De Maeyer and A. De Wulf, (2011). Accuracy assessment of a LiDAR digital terrain model by using RTK GPS and total station. EARSeL eProceedings 10(8): 1 - 8.

Stempfhuber, W., (2013). 3D - RTK capability of single GNSS receivers. International Archives of the Photogrammetry, Remote Sensing and Spatial Information Sciences 40: 379 - 384.

Võ Chí Mỹ, Robert Dudek, (2015). Nghiên cứu khả năng ứng dụng máy bay không người lái trong công tác trắc địa mỏ và giám sát môi trường mỏ. Tuyển tập Hội nghị khoa học và Công nghệ mỏ Việt Nam: Công nghiệp mỏ Việt Nam - cơ hội và thách thức, Vũng Tàu.

Vũ Phan Long and Lê Thắng, (2014). Thử nghiệm thiết bị bay không người lái thành lập bản dồ 3D hành lang tuyến điện. Hội nghị khoa học ngành Địa hình quân sự.

Xí nghiệp bay chụng ảnh hàng không, (2011). Báo cáo kết quả thử nghiệm xử lý ảnh chụp từ thiết bị không người lái MD - 1000. Hội nghị khoa học ngành Địa hình quân sự. Việt Nam. 